\title{
MMW-ONLINE-SPRECHSTUNDE
}

\author{
Kompetente Antworten innerhalb von 48 Stunden! \\ Unsere Experten beantworten medizinische \\ Fragen, die sich in Ihrem Praxisalltag ergeben.
}
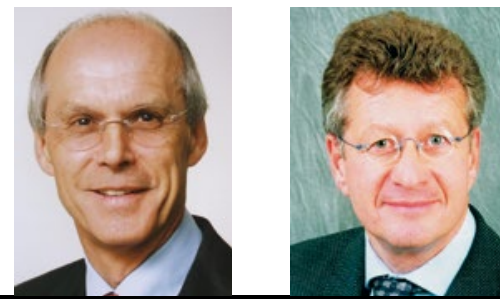

\section{Sollten Sportler Magnesium einnehmen?}

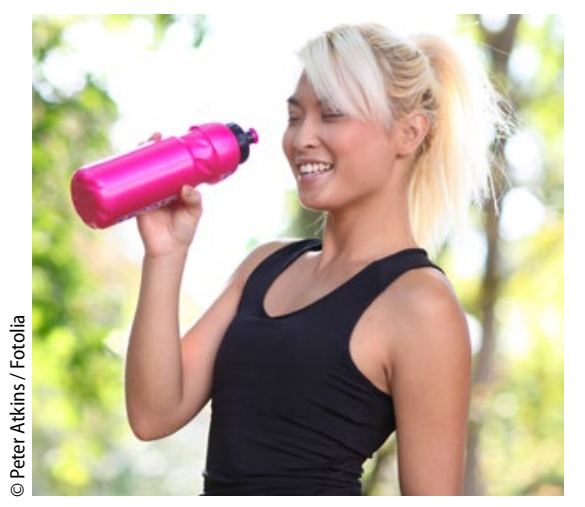

Magnesiumhaltige Sportgetränke sind trotz fehlender Evidenz beliebt.
? Anonymer Nutzer: Ist eine Magnesiumgabe für engagierte Freizeitsportler sinnvoll? Wenn ja, in welcher Dosis?

g

MMW-Experte Dr. Stiefelhagen: Personen, die regelmäßig Ausdauersport betreiben, verlieren vermehrt Magnesium über den Schweiß. Insgesamt benötigen sie ca. $500 \mathrm{mg}$ Magnesium täglich - das ist doppelt so viel wie jemand, der keinen Sport treibt. Daher sollten Sportler zusätzlich ca. $200 \mathrm{mg}$ Magnesium substituieren.

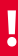
MMW-Experte Prof. FüeßI: Solide wissenschaftliche Daten, ob eine Substitution von Magnesium bei Leistungssportlern oder auch nur nächtlichen Wadenkrämpfen wirksam ist, gibt es leider nicht - dafür umso mehr persönliche Äußerungen im Netz. Der Mythos von Magnesium ist tief verwurzelt und würde wohl auch von wissenschaftlichen Untersuchungen nicht erschüttert werden. Solche können wir angesichts der guten Verträglichkeit, fehlender Alternativen und vor allem des geringen Preises aber auch kaum erwarten.

\section{Kassenleistung oder nicht?}

\section{Pneumokokken-Impfung nach Splenektomie}

$?$ Nutzerin „Kassandra“: Bei splenektomierten Patienten muss eine Pneumokokken-Impfung gemacht werden. Wird diese alle fünf Jahre aufgefrischt? Und ist das dann eine Kassenleistung? STIKO empfiehlt für Personen ab 60 Jahre eine einmalige Impfung mit dem Pneumokokken-Polysaccharid-Impfstoff. Bei Patienten mit angeborenen oder er- worbenen Immundefekten wird eine Auffrischimpfung nach fünf Jahren empfohlen. Dazu gehört sicherlich auch ein Zustand nach Splenektomie, sodass diese Auffrischimpfung auch von den Krankenkassen bezahlt werden müsste. MMW-Experte Prof. FüeßI: Nach den Empfehlungen der STIKO wird bei Patienten mit Zustand nach Splenektomie eine Grundimmunisierung mit dem 13-valenten Pneumokokken-Kon- jugatimpfstoff (Prevenar 13 ${ }^{\circledR}$ ) und nach 2-6 Monaten eine erste Auffrischimpfung mit dem 23-valenten Pneumokokken-Polysaccharidimpfstoff (Pneumo$\operatorname{vax} 23^{\oplus}$ ) empfohlen. Nach fünf Jahren sollte erneut mit PSV-23 aufgefrischt werden. Diese Impfungen werden von den Krankenkassen übernommen. Ausführliche Hinweise zu Impfungen und anderen Problemen von Patienten mit Asplenie finden sich im Internet unter http://asplenie-net.org. 